\title{
Blood culture contamination in the departments of paediatrics and child health at two tertiary training hospitals in central South Africa
}

\author{
R C Krause, $\mathrm{MB} \mathrm{ChB}$ (D) \\ Department of Paediatrics and Child Health, Faculty of Health Sciences, University of the Free State, Bloemfontein, South Africa
}

Corresponding author: R C Krause (rckrause6@gmail.com)

Background. Bloodstream infections are an important cause of mortality in children. Blood cultures (BCs) remain the primary means of identifying organisms and their antibiotic susceptibility profiles. A shortcoming of BCs is that up to $56 \%$ of positive cultures will represent contaminants. Poor adherence to standard practices applicable to BC sampling could explain an unacceptable contamination rate.

Objectives. To determine: (i) the BC contamination rate in the departments of paediatrics and child health at two tertiary hospitals in central South Africa; and (ii) BC sampling practices among paediatric clinicians.

Methods. The author determined the prevalence of BC contamination by analysis of laboratory data for the period 1 May - 27 August 2019 , and assessed possible factors contributing to BC contamination by surveying paediatric medical staff with a self-administered BC practices questionnaire.

Results. Of the 244 BCs reviewed, $25.4 \%$ were positive. The most commonly isolated pathogens were coagulase-negative staphylococci (CoNS) (33.3\%), Escherichia coli (22.2\%), Enterococcus faecium (16.7\%) and Acinetobacter baumannii (11.1\%). In total, 15.2\% of the BCs yielded contaminants and $2.9 \%$ had polymicrobial growth. The most common contaminant was CoNS. Approximately $68 \%$ of clinicians were not aware of BC sampling guidelines, and even among those who were aware of the guidelines, non-compliance was reported.

Conclusions. The $\mathrm{BC}$ contamination rate was higher than internationally accepted rates. Educating clinicians on specific BC sampling guidelines is strongly recommended to decrease the high rate of contamination observed in this study.

S Afr Med J 2022;112(2):102-107. https://doi.org/10.7196/SAMJ.2022.v112i2.16009

Globally, severe sepsis and septic shock is one of the most important risk factors for mortality in children. ${ }^{[1]}$ Statistics South Africa reported that the global trend was also relevant to South Africa (SA), with an estimated $20 \%$ of child deaths attributable to sepsis in $2013-2015 .^{[2]}$ Despite advances in molecular techniques and biomarkers, blood cultures (BCs) remain the primary means of identifying causative organisms of sepsis and determining the antimicrobial susceptibility of these organisms. ${ }^{[3-5]} \mathrm{BCs}$ guide physicians to use appropriate antibiotics and de-escalate from broad-spectrum antibiotics to specific antibiotic therapy, which forms the cornerstone of antibiotic stewardship. For this reason, BCs are essential in the highquality management of sepsis. ${ }^{[6,7]}$ The World Health Organization currently recognises $\mathrm{BC}$ as a priority specimen for the surveillance of antimicrobial resistance because of its clinical importance and accurate and uncomplicated methods of detection. ${ }^{[8]}$

$\mathrm{BC}$ is a common investigation performed in medical microbiology laboratories. A culture medium that supports the growth of most medically important micro-organisms is inoculated with peripheral blood obtained from the patient with clinically suspected sepsis. BC bottles that flag positive during incubation have fluid aspirated for Gram staining, microscopy and inoculation of appropriate media for incubation. Specific standards related to blood sampling techniques have been developed by various international infectious diseases societies and are endorsed by the South African Society of Clinical Microbiology (SASCM). These standards are outlined in Table $1 .{ }^{[9]}$

Despite the fact that one in five children in SA die as a result of sepsis, ${ }^{[2]}$ data on the causative organisms and prevalence of sepsis in children in SA are limited. ${ }^{[10,11]}$ To the author's knowledge, no previous studies on the $\mathrm{BC}$ contamination rate and $\mathrm{BC}$ sampling methods have been conducted in the departments of paediatrics and child health at two tertiary training hospitals (Pelonomi and Universitas hospitals) in central SA. Reports in the literature on SA research regarding paediatric sepsis, $\mathrm{BC}$ sampling techniques and contamination rates are scarce. ${ }^{[12]}$

\section{Objectives}

To assess the $\mathrm{BC}$ contamination rate and determine $\mathrm{BC}$ sampling practices among clinicians in the departments of paediatrics and child health at two tertiary training hospitals associated with the University of the Free State in Bloemfontein, SA.

\section{Methods}

A descriptive study was conducted in the departments of paediatrics and child health at Pelonomi and Universitas hospitals. Pelonomi Tertiary Hospital is a tertiary-level hospital in Free State Province with two general paediatric wards, an acute diarrhoea and severe acute malnutrition ward, and a paediatric intensive care unit (PICU). Universitas Academic Hospital is a tertiary hospital that receives referrals from secondary hospitals in the Free State and Northern Cape provinces of SA and the neighbouring country of Lesotho. Universitas Academic Hospital has a general paediatric ward, a paediatric cardiology high-care unit, a paediatric oncology ward and a PICU. Neonatal units at both hospitals and BCs performed on neonatal patients ( $<28$ days after birth) in the paediatric wards were excluded from the study. Paediatric surgery and orthopaedic patients were also excluded.

The BC contamination rate for the period 1 May - 27 August 2019 was determined by accessing laboratory data of the National Health 


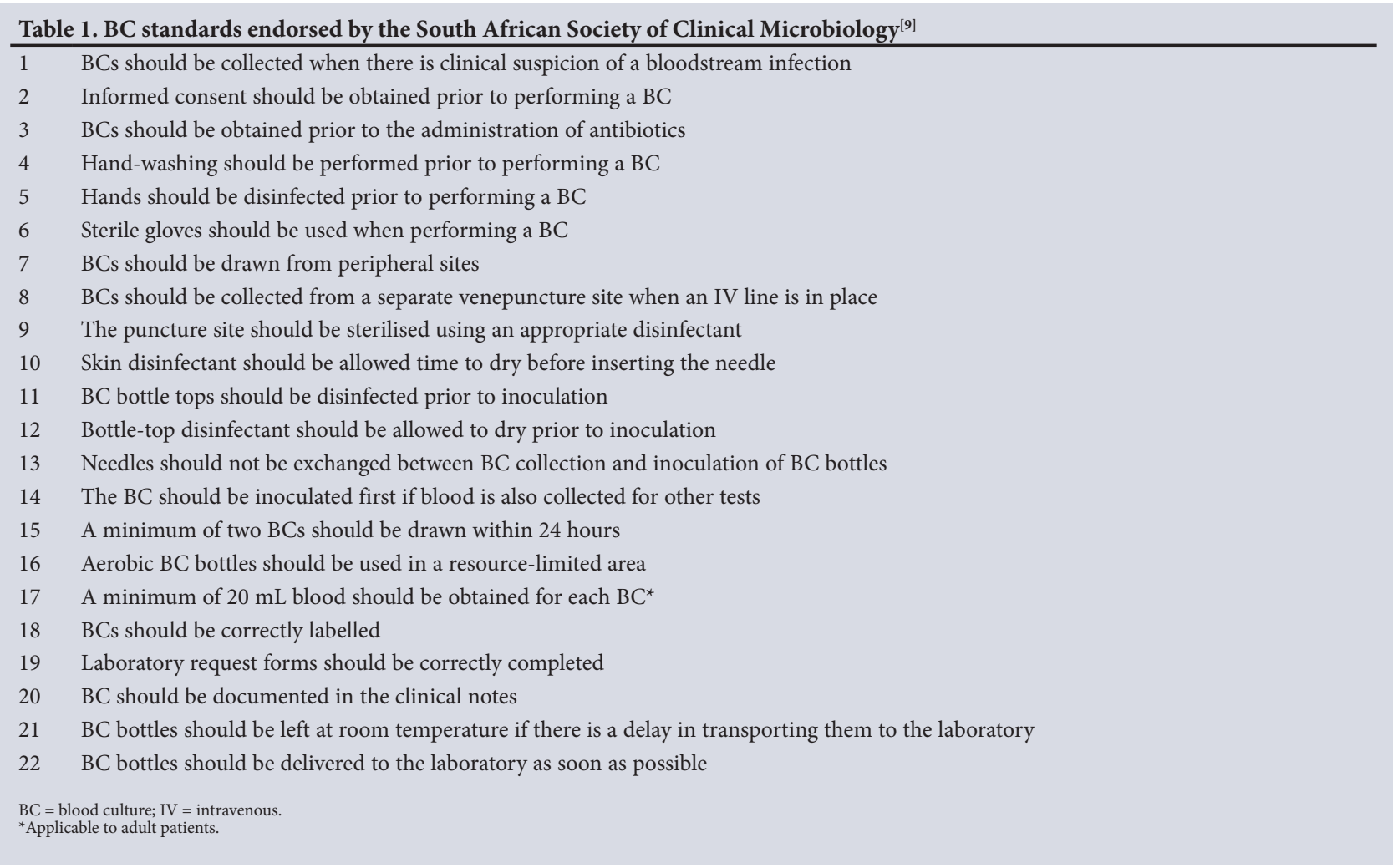

Laboratory Service (NHLS). All BCs submitted for every patient who was admitted to paediatric wards were included in the study. The clinical relevance of any organism isolated from a $\mathrm{BC}$ should be interpreted in the context of the patient's presenting illness, underlying conditions and recent procedures and/or devices present. In cases where organisms traditionally labelled as contaminants (coagulasenegative staphylococci (CoNS), Corynebacterium species, Bacillus species, Clostridium perfringens, Micrococcus species and viridans streptococci) were cultured, these cultures were only regarded as clinically relevant if the organism was isolated from two different specimens. This approach was based on the fact that the odds of having contaminated both cultures with the same organism are low. ${ }^{[5]}$

Clinicians' BC sampling practices were determined with an anonymous questionnaire completed by doctors working in the departments of paediatrics and child health during the study period. The questionnaire was broadly based on the guideline on $\mathrm{BC}$ sampling standards endorsed by the SASCM, as summarised in Table $1 .{ }^{[9]}$ Information obtained with the questionnaire included the participants' level of employment, and whether they had been aware of the SASCM guideline pertaining to BCs and had received previous teaching/training on $\mathrm{BC}$ sampling techniques. Regarding techniquespecific practices, they were asked to indicate the frequency at which the standards had been applied, ranging from always to never.

At Pelonomi and Universitas hospitals, BCs are collected by clinicians and not nursing staff. Only clinicians who had taken BCs during the study period were eligible to complete an anonymous questionnaire. The clinicians referred to in this study were consultants, registrars, medical officers and intern doctors. The specific time frame 1 May - 27 August 2019 was selected to coincide with the intern doctors' 4-monthly rotation through the departments of paediatrics and child health at the two hospitals.

Data were analysed using Excel 2010 spreadsheets (Microsoft Corp., USA) and QI Macros 2020 (KnowWare International Inc., USA) add-in for Excel.
Prior to data collection, approval for the research was obtained from the Health Sciences Research Ethics Committee of the Faculty of Health Sciences, University of the Free State (ref. no. UFSHSD2019/0377/0110). Permission for the research was also obtained from the Free State Province Department of Health and the NHLS. No patient consent was required, as only laboratory data were accessed. Written informed consent was obtained from the participating clinicians before completion of the questionnaire. Participation was anonymous and no personal information was recorded on the questionnaire.

\section{Results}

A total of 350 BCs were performed over a period of 4 months in the departments of paediatrics and child health of Pelonomi and Universitas hospitals, of which 244 (69.7\%) were included in this study. Of the $106 \mathrm{BCs}$ that were excluded, 2 lacked information on the patient's age, 76 were obtained from patients $<28$ days old, and 28 were performed on paediatric patients admitted to wards other than the wards specified in this study (e.g. paediatric surgery wards).

Only one BC bottle per patient was submitted to the laboratory in all 244 cases. Fifty-seven (23.4\%) of the BCs were sampled at Pelonomi Hospital and the remaining $187(76.6 \%)$ at Universitas Hospital. Table 2 lists the organisms isolated from the BCs, excluding the organisms present in the polymicrobial cultures. Table 3 summarises the BC findings. The rate of positive BCs was $25.4 \%(n=62)$, and of these isolates, 18 were pathogenic organisms. The results differed notably between the two hospitals, with 11 (19.3\%) of the BCs performed at Pelonomi Hospital yielding positive growth compared with $27.3 \%(n=51)$ of those at Universitas Hospital. Contaminants were isolated from $14.0 \%(n=8)$ of the BCs performed at Pelonomi Hospital and $15.5 \%(n=29)$ of those at Universitas Hospital. The overall contamination rate was $15.2 \%$.

In total, $135 \mathrm{BCs}$ in this study (55.3\%) were from children aged $\geq 28$ days to $<24$ months, of which 27 specimens (20.0\%) were 
Table 2. Laboratory findings of BCs obtained from paediatric patients

\begin{tabular}{|c|c|c|c|}
\hline \multirow[b]{2}{*}{ BC findings } & \multicolumn{2}{|c|}{ Hospital } & \multirow[b]{2}{*}{ Total $(N=244), n(\%)$} \\
\hline & Pelonomi $(N=57), n(\%)$ & Universitas $(N=187), n(\%)$ & \\
\hline Positive & $11(19.3)$ & $51(27.3)$ & $62(25.4)$ \\
\hline Pathogenic organism isolated & $2(3.5)$ & $16(8.6)$ & $18(7.4)$ \\
\hline Contaminants & $8(14.0)$ & $29(15.5)$ & $37(15.2)$ \\
\hline Polymicrobial growth & $1(1.8)$ & $6(3.2)$ & $7(2.9)$ \\
\hline No growth & $46(80.7)$ & $136(72.7)$ & $182(74.6)$ \\
\hline
\end{tabular}

\begin{tabular}{ll}
\multicolumn{2}{l}{ Table 3. Micro-organisms isolated from paediatric BCs } \\
\hline Organisms isolated & $\boldsymbol{n}(\%)$ \\
\hline Pathogens (N=18) & \\
$\quad$ Acinetobacter baumannii & $2(11.1)$ \\
CoNS* & $6(33.3)$ \\
Enterococcus faecium & $3(16.7)$ \\
Escherichia coli & $4(22.2)$ \\
Klebsiella pneumoniae & $1(5.6)$ \\
Pseudomonas aeruginosa & $1(5.6)$ \\
Streptococcus pneumoniae & $1(5.6)$ \\
Contaminants (N=37) & \\
CoNS & $31(83.8)$ \\
Corynebacterium species & $2(5.4)$ \\
Micrococcus species & $1(2.7)$ \\
Pantoea species & $1(2.7)$ \\
Streptococcus mitis & $2(5.4)$ \\
BC = blood culture; CoNS = coagulase-negative staphylococci. & \\
*Present in two separate BCs. &
\end{tabular}

contaminated. Of all the contaminated BCs $(n=37), 27$ (73.0\%) originated from this age group.

Of the 42 questionnaires handed out, 34 were completed, giving a response rate of $81.0 \%$. Six $(17.6 \%)$ of the participants were consultants, $17(50.0 \%)$ were registrars, $2(5.9 \%)$ were medical officers and 9 (26.5\%) were interns. As illustrated in Fig. 1, BC sampling practices varied widely between the participants, with 15 (44.1\%) indicating that they never obtained verbal consent before collecting the specimen. Three participants (8.8\%) either sometimes or never washed their hands before performing the procedure, while 9 (26.5) rarely or never left the disinfectant on the patient's skin or the $\mathrm{BC}$ bottle tops to dry before drawing blood and inoculating the culture medium.

Table 4 summarises the participants' knowledge and practices regarding $\mathrm{BCs}$ and sampling techniques. Only 11 (32.4\%) of the participants indicated that they were aware of the SASCM guideline for BC specimen collection. Of the 8 participants (23.5\%) who reported having received a lecture on the correct collection technique of BCs since starting to work in the department of paediatrics and child health, 3 were consultants, 4 were registrars and 1 was an intern. Three participants (8.8\%) did not keep the specimens at room temperature when transport to the laboratory was delayed, 12 (35.3\%) did not apply aseptic techniques on days that were busier than normal, and $12(35.3 \%)$ repalpated the vein after disinfecting the patient's skin. Chlorhexidine was used by 18 participants (52.9\%) when disinfecting the venepuncture site. Overall analysis of the questionnaire results showed that only 1 (2.9\%) of the 34 participants responded in a way suggesting complete compliance with the 22 standards of the SASCM guideline on BC sampling.

\section{Discussion}

An alarming finding from this study was the $\mathrm{BC}$ contamination rate of $15 \%$, approximately three times the contamination rate at other SA public sector hospitals. ${ }^{[7,13]}$ Internationally, acceptable BC contamination rates are $\leq 3 \% .{ }^{[14]}$ Factors contributing to this high contamination rate could include poor compliance with $\mathrm{BC}$ sampling standards, such as hand hygiene practices and inadequate skin and BC bottle-top preparation, lack of use of sterile gloves (as shown in Fig. 1), and unavailability of sterile gloves. ${ }^{[7]}$ Adverse events related purely to a high $\mathrm{BC}$ contamination rate include (but are not limited to): (i) an increased hospitalisation time by 1 - 5 days; (ii) an associated 20 $39 \%$ increase in hospital fees; (iii) an $80 \%$ increase in microbiology laboratory charges; and (iv) a $40 \%$ increase in unnecessary antibiotic use with a resultant increase in antibiotic resistance, and antibioticassociated side-effects, including allergic drug reactions. ${ }^{[5,7,15]}$

The contamination rate of $20 \%$ found among BCs from children aged $<24$ months, which represented $73.0 \%$ of all the contaminated specimens, could be attributed to the technical difficulty of drawing blood from a child. Children this young are unable to co-operate, because of a lack of understanding and the pain and trauma experienced from the procedure. It is clear that this BC contamination rate needs to be addressed, not only to avoid unnecessary expenses in a resource-limited setting, but also to prevent adverse patient events. El Feghaly et al. ${ }^{[15]}$ made the statement that ' $[\mathrm{b}]$ lood culture contamination is a safety and quality concern in children's hospitals'.

The 7 (2.9\%) polymicrobial BC results were excluded from the data analysis, as obtaining clinical information regarding patients was beyond the scope of the study. In a setting where the BC contamination rate is five times greater than the international norm, it is almost impossible to differentiate between overt BC contamination and true polymicrobial septicaemia without clinical information. Polymicrobial septicaemia most commonly occurs in patients who were admitted to hospital within the preceding 90 days, patients with malignancies, patients who previously had central venous lines, and immunocompromised individuals. ${ }^{[9]}$ In a systematic review, Reddy et al. ${ }^{[10]}$ reported that $1.2 \%$ of bloodstream infections in African countries were due to polymicrobial organisms. At both the study hospitals this rate was found to be higher at $~ 3 \%$, which might have been secondary to the high $\mathrm{BC}$ contamination rate. However, this argument may be purely speculative.

CoNS were classified as pathogenic when isolated from two different BCs from the same patient. ${ }^{[9]}$ CoNS were regarded as pathogenic (i.e. 3 CoNS bloodstream infection episodes) in $6(2.4 \%)$ of the 244 BCs that were analysed and classified as contaminants in 31 (12.7\%). In all 6 cases where CoNS were pathogenic, the patients were $<24$ months of age. In a district hospital in Western Cape Province, SA, Abrahams et al. ${ }^{[7]}$ found that the contamination rate due to CoNS was only $3 \%$. This difference may be due to dissimilarities between the two study populations, or to variations in sampling practices. 


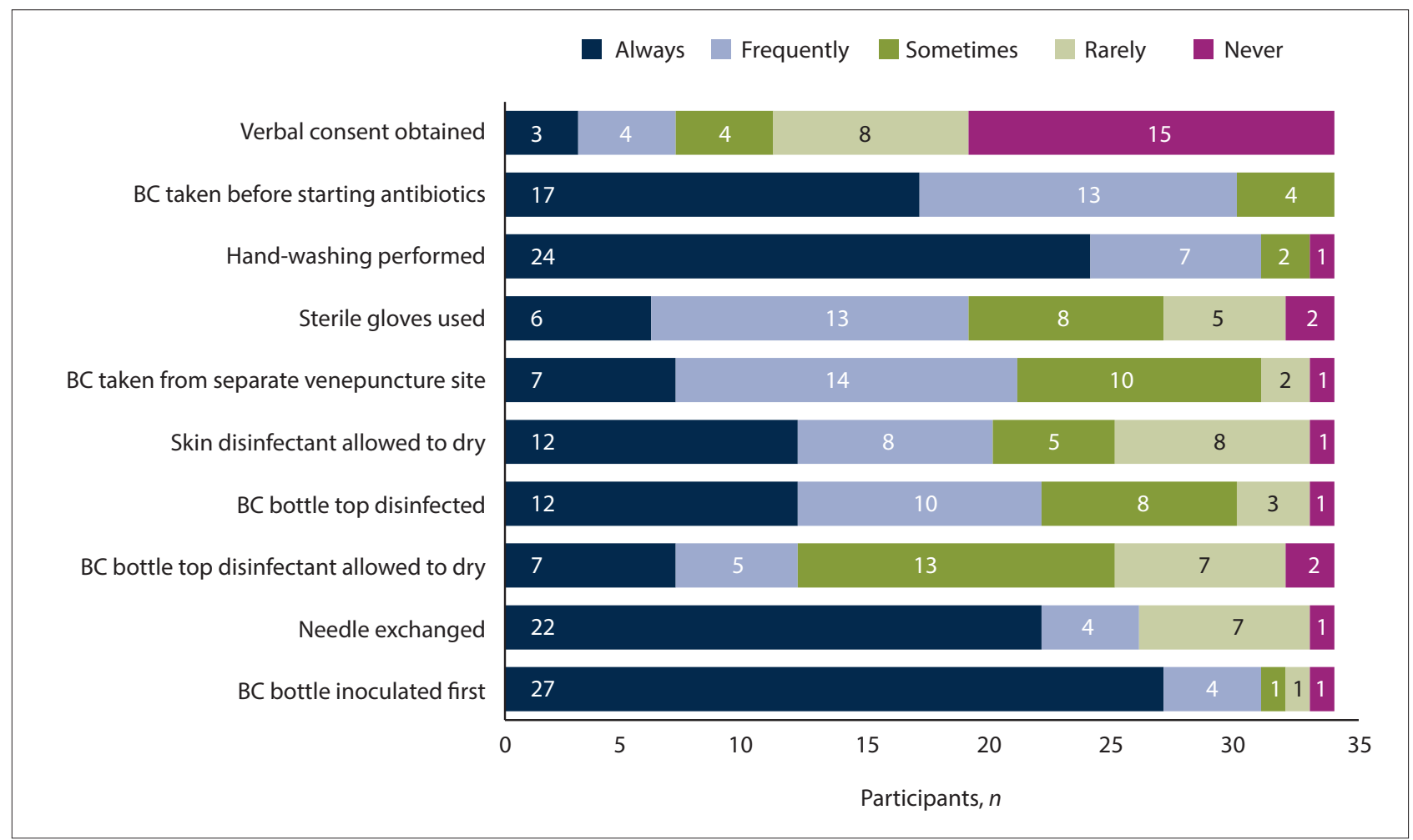

Fig. 1. Clinicians' adherence to the South African Society for Clinical Microbiology guidelines for collecting BCs ( $\mathrm{N}=34$ respondents). (BC $=$ blood culture.)

The SA standards on BC sampling propose using alcohol or povidone iodine to disinfect the skin at the venepuncture site. ${ }^{[9]}$ At Pelonomi and Universitas hospitals, chlorhexidine with alcohol or alcohol swabs are readily available and have therefore been used by the participants in the questionnaire survey. A recent meta-analysis reported no significant difference between chlorhexidine-alcohol and povidone iodine solutions for skin antisepsis. ${ }^{[10]} \mathrm{A}$ major problem with the use of alcohol swabs is that most are far too small to clean the skin surface area adequately, and the swabs dry out very quickly. For this reason, the proposed BC packs with cotton wool swabs and 70\% alcohol or chlorhexidine gluconate in alcohol are preferable. Ombelet et al. ${ }^{[5]}$ described a technique where alcohol swabs were used multiple times until dirt was removed from a patient's skin, and only then was a different antiseptic agent applied to the venepuncture site. Sixteen $(47.1 \%)$ of the 34 participants in this study used alcohol swabs for skin antisepsis.

Although $30(88.2 \%)$ of the participants indicated that they used $1-2 \mathrm{~mL}$ of blood when inoculating BC bottles, $<50 \%(n=13 ; 43.3 \%)$ of this subgroup indicated that they always used this blood volume. The remaining 14 (46.7\%) frequently inoculated the BC bottles with $1-2 \mathrm{~mL}$ of blood, and $3(10.0 \%)$ sometimes. In a quality improvement initiative study by El Feghaly et al.., ${ }^{[15]}$ it was noted that nursing staff routinely sampled $1-3 \mathrm{~mL}$ of blood, irrespective of a patient's body weight. They reported that this specific volume of blood was used because no optimised blood volume charts were available in their setting. ${ }^{[15]}$ This situation also applies in our setting and possibly explains the varying frequency at which clinicians sampled different blood volumes for paediatric BCs. Multiple authors agree that by optimising the blood volume inoculated into $\mathrm{BC}$ bottles, the $\mathrm{BC}$ yield is significantly improved. ${ }^{[4,11,15,16]}$

Despite roughly a third of participants indicating that they were aware of the SASCM guideline on BC sampling, only one participant completed the questionnaire in a manner reflecting compliance with this guideline. This finding emphasises the importance of improving clinicians' knowledge and skills pertaining to the correct sampling techniques required when performing $\mathrm{BCs}$ on paediatric patients.

In this study, we attempted to evaluate $\mathrm{BC}$ sampling practices and not only the $\mathrm{BC}$ contamination rate. We differentiated between results regarding contaminants and pathogens and identified errors in BC sampling practices. The limitations of our study were that we did not evaluate: $(i)$ the availability of proper equipment for performing BCs; (ii) the clinical indications for BC sampling; (iii) whether contaminant organisms were treated as pathogens on clinical grounds or not; (iv) BC request forms; (v) sites from which BCs were sampled; ( $v i$ ) the volume of blood inoculated in each $\mathrm{BC}$ bottle; and (vii) the timing of antibiotic administration and $\mathrm{BC}$ sampling; while (viii) $\mathrm{BC}$ practices were self-reported and not directly observed.

\section{Recommendations}

The following recommendations are proposed to improve specimen collection techniques when performing BCs on paediatric patients:

- Pictorial wall charts should be put up in procedure rooms in paediatric wards at Pelonomi and Universitas hospitals, stressing the importance of correct BC sampling techniques.

- In younger children, assistance with restraining the child while taking the specimen may help to decrease the $\mathrm{BC}$ contamination rate.

- Weight-based BC volume charts should also be put up in the procedure rooms in paediatric wards.

- Weekly antibiotic stewardship rounds should serve as an opportunity to educate clinicians on the best practice when sampling $\mathrm{BC}$ specimens.

- Quarterly refreshing of BC sampling guidelines and techniques for all clinicians working in the paediatric departments of Pelonomi and Universitas hospitals should be considered.

- Prepacked BC kits and procedural checklists should be used.

- Standard-sized small alcohol swabs in prepacked BC kits should preferably be replaced with cotton wool swabs and $70 \%$ alcohol 
Table 4. Participants' knowledge and practices regarding BCs

\begin{tabular}{|c|c|}
\hline Questionnaire item & $n(\%)$ \\
\hline \multicolumn{2}{|c|}{ Are you aware of the South African Society of Clinical Microbiology guideline for BC specimen collection? } \\
\hline Yes & $11(32.4)$ \\
\hline No & $23(67.6)$ \\
\hline \multicolumn{2}{|c|}{$\begin{array}{l}\text { Since starting to work in the Department of Paediatrics and Child Health, have you ever had a lecture about } \\
\text { the importance of BCs? }\end{array}$} \\
\hline Yes & $8(23.5)$ \\
\hline No & $26(76.5)$ \\
\hline \multicolumn{2}{|c|}{$\begin{array}{l}\text { Since starting to work in the Department of Paediatrics, have you ever had a lecture about the correct collection } \\
\text { technique of BCs? }\end{array}$} \\
\hline Yes & $8(23.5)$ \\
\hline No & $26(76.5)$ \\
\hline \multicolumn{2}{|c|}{ When there is a delay in $\mathrm{BC}$ transport to laboratory, do you leave the $\mathrm{BC}$ bottle at room temperature? } \\
\hline Yes & $31(91.2)$ \\
\hline No & $3(8.8)$ \\
\hline \multicolumn{2}{|c|}{ What volume of blood do you inoculate into paediatric $\mathrm{BC}$ bottles? } \\
\hline$<1 \mathrm{~mL}$ & $1(2.9)$ \\
\hline $1-2 \mathrm{~mL}$ & $30(88.2)$ \\
\hline$>2 \mathrm{~mL}$ & $3(8.8)$ \\
\hline \multicolumn{2}{|c|}{ What antiseptic do you use when disinfecting the venepuncture site? } \\
\hline Chlorhexidine & $18(52.9)$ \\
\hline Povidone tincture & 0 \\
\hline Alcohol swabs & $16(47.1)$ \\
\hline Iodine & 0 \\
\hline \multicolumn{2}{|c|}{$\begin{array}{l}\text { When a patient presents with a clinically diagnosable focus of infection and is being admitted to hospital, } \\
\text { is it necessary to do a BC? }\end{array}$} \\
\hline Yes & $29(85.3)$ \\
\hline No & $5(14.7)$ \\
\hline \multicolumn{2}{|c|}{$\begin{array}{l}\text { With specific reference to unco-operative patients, do you ask for assistance to aid in restraining the patient } \\
\text { while collecting a BC sample? }\end{array}$} \\
\hline Yes & $31(91.2)$ \\
\hline No & $3(8.8)$ \\
\hline \multicolumn{2}{|c|}{ On a day that is busier than normal, do you still observe aseptic technique while collecting a BC? } \\
\hline Yes & $22(64.7)$ \\
\hline No & $12(35.3)$ \\
\hline \multicolumn{2}{|c|}{ After disinfecting a patient's skin, do you repalpate the vein? } \\
\hline Yes & $12(35.3)$ \\
\hline No & $22(65.7)$ \\
\hline
\end{tabular}

or chlorhexidine gluconate in alcohol to ascertain adequate skin antisepsis.

- Quarterly review of the BC contamination rate at these institutions should be monitored following the implementation of these recommendations, and adherence to set targets (e.g. $<5 \%$ or $<3 \%$ ) should be achieved.

- Future studies need to be conducted in these institutions to assess blood volume and $\mathrm{BC}$ yield and the clinical indications for $\mathrm{BC}$ sampling.

\section{Conclusions}

We found a $\mathrm{BC}$ contamination rate that was substantially higher than internationally accepted rates. We also demonstrated that clinicians were not aware of BC sampling guidelines. Among the $32.4 \%$ of clinicians who were aware of these guidelines, compliance was not observed, with only one participant's questionnaire results indicating adherence to the guidelines.
Declaration. The research for this study was done in partial fulfilment of the requirements for RCK's MMed (Paediatrics) degree at the University of the Free State.

Acknowledgements. The author thanks the clinicians at Pelonomi and Universitas hospitals who participated in the questionnaire survey, and Dr Daleen Struwig, medical writer/editor, Faculty of Health Sciences, University of the Free State, for technical and editorial preparation of the manuscript.

Author contributions. Sole author.

Funding. None.

Conflicts of interest. None.

1. Mangia CMF, Kissoon N, Carcillo JA. Sepsis and septic shock: A global overview. J Pediatr Infect Dis 2009;4(2):71-76. https://doi.org/10.3233//PI-2009-015

2. Statistics South Africa. Mortality and causes of death in South Africa, 2015: Findings from death Statistics South Africa. Mortality and causes of death in South Africa, 2015: Findings from death
notification. Statistical release P0309.3. 28 February 2017. http://www.statssa.gov.za/publications/ P03093/P030932015.pdf (accessed 5 August 2021). 
3. Dien Bard J, McElvania TeKippe E. Diagnosis of bloodstream infections in children. J Clin Microbiol 2016;54(6):1418-1424. https://doi.org/10.1128/JCM.02919-15

4. Buttery JP. Blood cultures in newborns and children: Optimising an everyday test. Arch Dis Child Fetal Neonatal Ed 2002;87(1):F25-F28. https://doi.org/10.1136/fn.87.1.F25

5. Ombelet S, Barbé B, Affolabi D, et al. Best practices of blood cultures in low- and middle-income countries. Front Med 2019;6:131. https://doi.org/10.3389/fmed.2019.00131

6. Rhodes A, Evans LE, Alhazzani W, et al. Surviving Sepsis Campaign: International guidelines for management of sepsis and septic shock: 2016. Intensive Care Med 2017;43(3):304-377. https://doi org/10.1007/s00134-017-4683-6

7. Abrahams MS, Whitelaw AC, Orth H. Time for a culture change? Suboptimal compliance with blood culture standards at a district hospital in Cape Town. S Afr Med J 2015;105(12):1039-1043. https://doi. org/10.7196/SAMJ.2015.v105i12.9442

8. World Health Organization. Global Antimicrobial Resistance Surveillance System: Manual for early implementation. Geneva: WHO, 2015. https://apps.who.int/iris/bitstream/ handle/10665/188783/9789241549400_eng.pdf (accessed 5 August 2021).

9. Ntusi N, Aubin L, Oliver S, et al. Guideline for the optimal use of blood cultures. S Afr Med J No10:100(12):839-843. 10. Reddy EA, Shaw AV, Crump JA. Community-acquired bloodstream infections in Africa: A systematic
review and meta-analysis. Lancet Infect Dis 2010;10(6):417-432. https://doi.org/10.1016/S1473review and meta-analysis. Lancet Infect Dis 2010;10(6):417-432. https://doi.org/10.1016/S1473-
$3099(10) 70072-4$ 3099(10)70072-4

11. Lochan H, Pillay V, Bamford C, et al. Bloodstream infections at a tertiary level paediatric hospital in South Africa. BMC Infect Dis 2017;17(1):750. https://doi.org/10.1186/s12879-017-2862-2
12. Dramowski A, Cotton MF, Rabie H, Whitelaw A. Trends in paediatric bloodstream infections at a South African referral hospital. BMC Pediatr 2015;15(1):33. https://doi.org/10.1186/s12887-015-

13. Crichton $\mathrm{H}$, O'Connell N, Rabie H, et al. Neonatal and paediatric bloodstream infections: Pathogens, antimicrobial resistance patterns and prescribing practice at Khayelitsha District Hospital, Cape Town, South Africa. S Afr Med J 2018;108(2):99-104. https://doi.org/10.7196/SAMJ.2017.v108i2.12601

14. Baron EJ, Miller JM, Weinstein MP, et al. Executive summary: A guide to utilisation of the microbiology laboratory for diagnosis of infectious diseases: 2013 recommendations by the Infectious Diseases Society of America (IDSA) and the American Society for Microbiology (ASM). Clin Infect Dis 2013;57(4):485-488. https://doi.org/10.1093/cid/cit441

15. El Feghaly RE, Chatterjee J, Dowdy K, et al. A quality improvement initiative: Reducing blood culture contamination in a children's hospital. Pediatrics 2018;142(4):e20180244. https://doi.org/10.1542/ peds.2018-0244

16. Lamy B, Dargère S, Arendrup MC, et al. How to optimise the use of blood cultures for the diagnosis of bloodstream infections? A state-of-the art. Front Microbiol 2016;7:697. https://doi.org/10.3389/ fmicb.2016.00697

Accepted 22 October 2021 